\title{
Functional inequalities on manifolds with non-convex boundary
}

\author{
Li-Juan Cheng ${ }^{1,2}$, Anton Thalmaier ${ }^{1} \&$ James Thompson ${ }^{1, *}$ \\ ${ }^{1}$ Mathematics Research Unit, FSTC, University of Luxembourg \\ Maison du Nombre, 6, Avenue de la Fonte, \\ 4364 Esch-sur-Alzette, Grand Duchy of Luxembourg; \\ ${ }^{2}$ Department of Applied Mathematics, Zhejiang University of Technology \\ Hangzhou 310023, The People's Republic of China
}

Email: lijuan.cheng@uni.lu,chenglj@zjut.edu.cn, anton.thalmaier@uni.lu,james.thompson@uni.lu

\begin{abstract}
In this article, new curvature conditions are introduced to establish functional inequalities including gradient estimates, Harnack inequalities and transportation-cost inequalities on manifolds with non-convex boundary.
\end{abstract}

Keywords Ricci curvature, gradient inequality, log-Sobolev inequality, geometric flow

MSC(2010) 60J60, 58J65, 53C44

Citation: L.-J. Cheng, A. Thalmaier and J. Thompson. Functional inequalities on manifolds with non-convex boundary. https://doi.org/10.1007/s11425-000-0000-0

\section{Introduction}

Let $(M, g)$ be a complete and connected Riemannian manifold of dimension $d \geqslant 2$, with Riemannian distance $\rho$, boundary $\partial M$ and inward pointing unit normal vector $N$. Define the second fundamental form of the boundary by

$$
\mathrm{II}(X, Y)=-\left\langle\nabla_{X} N, Y\right\rangle, \quad X, Y \in T_{x} \partial M, \quad x \in \partial M
$$

where $T \partial M$ denotes the tangent bundle of $\partial M$. In order to study non-convex boundaries we will perform a conformal change of metric such that the boundary is convex under the new metric. In particular, we will use the fact that if

$$
\mathscr{D}:=\left\{\phi \in C_{b}^{2}(M): \inf \phi=1, \mathrm{II} \geqslant-N \log \phi\right\}
$$

and $\phi \in \mathscr{D}$ then the boundary $\partial M$ is convex under the metric $\phi^{-2} g$ (see [16, Theorem 1.2.5]).

Given a $C^{1}$-vector field $Z$ on $M$, consider the elliptic operator $L:=\Delta+Z$ and let $X_{t}^{x}$ be a reflecting $L$-diffusion process starting from $X_{0}^{x}=x$. Then $X_{t}^{x}$ solves the Stratonovich equation

$$
\mathrm{d} X_{t}^{x}=\sqrt{2} u_{t}^{x} \circ \mathrm{d} B_{t}+Z\left(X_{t}^{x}\right) \mathrm{d} t+N\left(X_{t}^{x}\right) \mathrm{d} l_{t}^{x}, \quad X_{0}^{x}=x
$$

\footnotetext{
* Corresponding author
} 
where $u_{t}^{x}$ is the horizontal lift of $X_{t}^{x}$ to the orthonormal frame bundle $\mathrm{O}(M)$ with $\pi\left(u_{0}^{x}\right)=x, B_{t}$ is a standard $\mathbb{R}^{d}$-valued Brownian motion defined on a complete naturally filtered probability space $\left(\Omega,\left\{\mathscr{F}_{t}\right\}_{t \geqslant 0}, \mathbb{P}\right)$ and $l_{t}^{x}$ is a continuous adapted nondecreasing and nonnegative process which increases only on $\left\{t \geqslant 0: X_{t}^{x} \in \partial M\right\}$. The process $l_{t}^{x}$ is the local time of $X_{t}^{x}$ on $\partial M$.

We assume that $X_{t}^{x}$ is non-explosive for each $x \in M$. Then the diffusion process $X_{t}^{x}$ gives rise to the Neumann semigroup $P_{t}$ which solves the diffusion equation $\left(\partial_{t}-L\right) P_{t}=0$ with Neumann boundary condition $N P_{t}=0$. Furthermore $P_{t} f(x)=\mathbb{E}\left[f\left(X_{t}^{x}\right)\right]$ for each $f \in C_{b}(M)$.

In [7], Hsu found a probabilistic formula for $\nabla P_{t} f$ for compact manifolds with boundary, which he used to derive a gradient estimate. Feng-Yu Wang extended it to the non-compact case [16, Theorem 3.2.1] under the assumption that $|\nabla P . f|$ is uniformly bounded on $[0, t] \times M$. Wang's formula is given below by Theorem 2.1. In [16, Proposition3.2.7], he proved that if

$$
\operatorname{Ric}^{Z}:=\operatorname{Ric}-\nabla Z \geqslant K
$$

for some $K \in C(M)$ and if there exists $\phi \in \mathcal{D}$ such that

$$
\tilde{K}_{\phi}:=\inf _{M}\left\{\phi^{2} K+\frac{1}{2} L \phi^{2}-\left|\nabla \phi^{2}\right||Z|-(d-2)|\nabla \phi|^{2}\right\}>-\infty
$$

then $|\nabla P . f|$ is uniformly bounded on $[0, t] \times M$ by an expression involving the constant $\tilde{K}_{\phi}$.

In this article, we revisit this problem using coupling methods. In particular, we prove (see Theorem 2.2) that if there exists $\phi \in \mathcal{D}$ and a constant $K_{\phi}$ such that

$$
\operatorname{Ric}^{Z}+L \log \phi-2|\nabla \log \phi|^{2} \geqslant K_{\phi}
$$

then $|\nabla P . f|$ is uniformly bounded on $[0, t] \times M$ and our upper bound improves that of $[16$, Proposition 3.2.7]. We construct a suitable function $\phi$ in Proposition 3.3, under the assumption that there exist non-negative constants $\sigma$ and $\theta$ such that $-\sigma \leqslant \mathrm{II} \leqslant \theta$ and a positive constant $r_{0}$ such that on $\partial_{r_{0}} M:=$ $\left\{x \in M: \rho_{\partial}(x) \leqslant r_{0}\right\}$ the function $\rho_{\partial}$ is smooth, the norm of $Z$ is bounded and Sect $\leqslant k$ for some positive constant $k$.

F.-Y. Wang also considered Harnack and transportation-cost inequalities on manifolds with boundary [16]. We reconsider these problems too and find that the curvature conditions used to establish these inequalities can also be weakened and simplified. It is worth mentioning that we find a transportationcost inequality on the path space of the reflecting diffusion process which (see Theorem 2.8) recovers the results for the convex boundary case, making this aspect of the theory of functional inequalities on path space complete.

Let us now describe the organization of this article. In Section 2, we prove the gradient estimates, Harnack inequalities and transportation-cost inequalities for the Neumann semigroup via coupling methods. In Section 3, we construct a function $\phi$ which satisfies the new curvature conditions.

\section{Functional inequalities}

\subsection{Gradient estimates}

A derivative formula for $P_{t} f$ that does not involve derivatives of $f$ is typically called a Bismut formula $($ see $[4,5])$. The Bismut formula we introduce is of a type due originally to Thalmaier [9]. As mentioned in the introduction, Hsu [7] found this type of formula for compact manifolds with boundary. The following formula for manifolds with boundary, due to F.-Y. Wang [16, Theorem 3.2.1], does not require compactness. See also [1] for recent work on probabilistic representations of the derivative of Neumann semigroups.

Theorem 2.1. Let $t>0$ and $u_{0} \in \mathrm{O}_{x}(M)$ be fixed. Suppose $K \in C(M)$ and $\sigma \in C(\partial M)$ are such that $\operatorname{Ric}_{Z} \geqslant K$ and $\mathrm{II} \geqslant \sigma$. Assume that

$$
\sup _{s \in[0, t]} \mathbb{E}^{x}\left[\exp \left(-\int_{0}^{s} K\left(X_{r}\right) \mathrm{d} r-\int_{0}^{s} \sigma\left(X_{r}\right) \mathrm{d} l_{r}\right)\right]<\infty .
$$


Then there exists a progressively measurable process $\left\{Q_{s}\right\}_{s \in[0, t]}$ on $\mathbb{R}^{d} \otimes \mathbb{R}^{d}$ such that

$$
Q_{0}=I, \quad\left\|Q_{s}\right\| \leqslant \exp \left(-\int_{0}^{s} K\left(X_{r}\right) \mathrm{d} r-\int_{0}^{s} \sigma\left(X_{r}\right) \mathrm{d} l_{r}\right), \quad s \in[0, t]
$$

and for any $f \in C_{b}^{1}(M)$ such that $\nabla P . f$ is bounded on $[0, t] \times M$, for any $h \in C^{1}([0, t])$ with $h(0)=0$ and $h(t)=1$, we have

$$
u_{0}^{-1} \nabla P_{t} f(x)=\mathbb{E}^{x}\left[Q_{t} u_{t}^{-1} \nabla f\left(X_{t}\right)\right]=\frac{1}{\sqrt{2}} \mathbb{E}^{x}\left[f\left(X_{t}\right) \int_{0}^{t} \dot{h}(s) Q_{s} \mathrm{~d} B_{s}\right] .
$$

In order to use this formula it is necessary to check the uniform boundedness of $\nabla P . f$ on $[0, t] \times M$. In [16, Proposition 3.2.7], F.-Y. Wang did so using a conformal change of metric such that under the new metric the boundary is convex, and by then making a time change of the $L$-diffusion process $X_{t}$. Here, we use coupling methods to study this problem again and obtain improved upper bounds.

Theorem 2.2. If there exist $\phi \in \mathscr{D}$ and a constant $K_{\phi}$ such that

$$
\operatorname{Ric}^{Z}+L \log \phi-2|\nabla \log \phi|^{2} \geqslant K_{\phi}
$$

then for all $f \in C^{1}(M)$ such that $f$ is constant outside a compact set,

$$
\left|\nabla P_{t} f\right| \leqslant\|\phi\|_{\infty}\|\nabla f\|_{\infty} \mathrm{e}^{-K_{\phi} t}, \quad t>0 .
$$

Proof. We start with a conformal change of the metric $g$. Since $\phi \in \mathscr{D}$, the boundary $\partial M$ is convex under the metric $g^{\prime}:=\phi^{-2} g$. Let $\Delta^{\prime}$ and $\nabla^{\prime}$ be the Laplacian and gradient operator associated with the metric $g^{\prime}$. Then

$$
L=\phi^{-2}\left(\Delta^{\prime}+\phi^{2}(Z+(d-2) \nabla \log \phi)\right)=\phi^{-2}\left(\Delta^{\prime}+Z^{\prime}\right)
$$

where $Z^{\prime}:=\phi^{2}(Z+(d-2) \nabla \log \phi)$. For the process $X_{t}$ generated by $L$, viewed as a process on $\left(M, g^{\prime}\right)$, denoting by $\mathrm{d}_{I}$ the Itô differential, it follows that

$$
\mathrm{d}_{I} X_{t}=\sqrt{2} \phi^{-1}\left(X_{t}\right) u_{t} \mathrm{~d} B_{t}+\phi^{-2}\left(X_{t}\right) Z^{\prime}\left(X_{t}\right) \mathrm{d} t+N^{\prime}\left(X_{t}\right) \mathrm{d} l_{t}, \quad X_{0}=x
$$

where $B_{t}$ is the Brownian motion and the lift $u_{t}$ and boundary local time $l_{t}$ are defined now with respect to the metric $g^{\prime}$. Recall that in local coordinates, the Itô differential of a continuous semimartingale $X_{t}$ on $M$ is given (see [6] or [2]) by

$$
\left(\mathrm{d}_{I} X_{t}\right)^{k}=\mathrm{d} X_{t}^{k}+\frac{1}{2} \sum_{i, j=1}^{d} \Gamma_{i j}^{\prime k}\left(X_{t}\right) \mathrm{d}\left\langle X^{i}, X^{j}\right\rangle_{t}, \quad 1 \leqslant k \leqslant d
$$

where $\Gamma^{\prime k}$ are the Christoffel symbols of $g^{\prime}$. Similarly, let $Y_{t}$ solve

$$
\mathrm{d}_{I} Y_{t}=\sqrt{2} \phi^{-1}\left(Y_{t}\right)\left(1_{\left\{\left(X_{t}, Y_{t}\right) \notin \mathrm{cut}\right\}} P_{X_{t}, Y_{t}}^{\prime} u_{t} \mathrm{~d} B_{t}+1_{\left\{\left(X_{t}, Y_{t}\right) \in \operatorname{cut}\right\}} \tilde{u}_{t} \mathrm{~d} B_{t}^{\prime}\right)+\phi^{-2}\left(Y_{t}\right) Z^{\prime}\left(Y_{t}\right) \mathrm{d} t+N^{\prime}\left(Y_{t}\right) \mathrm{d} \tilde{l}_{t}
$$

with $Y_{0}=y$, lift $\tilde{u}_{t}$ and boundary local time $\tilde{l}_{t}$, where cut $\subset M \times M$ denotes the set of cut points and where $B_{t}^{\prime}$ is a Brownian motion independent of $B_{t}$ (see [16, Theorem 3.2.5] or [11, Section 2.1]). Now, for $(x, y) \notin$ cut and $x \neq y$, define

$$
I_{Z}^{\phi}(x, y):=\sum_{i=1}^{d}\left(U_{i}\right)^{2} \rho^{\prime}(x, y)+\left\langle\phi^{-2}(y) Z^{\prime}(y), \nabla^{\prime} \rho^{\prime}(x, \cdot)(y)\right\rangle^{\prime}+\left\langle\phi^{-2}(x) Z^{\prime}(x), \nabla^{\prime} \rho^{\prime}(\cdot, y)(x)\right\rangle^{\prime}
$$

where $\left\{U_{i}\right\}_{i=1}^{d}$ are vector fields on $M \times M$ such that $\nabla^{\prime} U_{i}(x, y)=0$ and

$$
U_{i}(x, y)=\left(\phi^{-1}(x) V_{i}, \phi^{-1}(y) P_{x, y}^{\prime} V_{i}\right), \quad 1 \leqslant i \leqslant d
$$


for $\left\{V_{i}\right\}_{i=1}^{d}$ a $g^{\prime}$-orthonormal basis of $T_{x} M$. Here $P_{x, y}^{\prime}$ denotes parallel displacement from $x$ to $y$ with respect to the metric $g^{\prime}$. Denote by $\rho^{\prime}$ the distance function for the metric $g^{\prime}$. Since the boundary $\partial M$ is convex under $g^{\prime}$, that is $\left.N^{\prime} \rho^{\prime}\right|_{\partial M} \leqslant 0$, by a slight modification of the proof of [11, Theorem 2.1.6], we have the following result which is similar to [16, Theorem 3.2.5]: if there exists $J \in C(M \times M)$ such that $J \geqslant I_{Z}^{\phi}$ outside the cut locus and $D(M)$, where $D(M):=\{(x, x): x \in M\}$, then

$$
\mathrm{d} \rho^{\prime}\left(X_{t}, Y_{t}\right) \leqslant \sqrt{2}\left(\phi^{-1}\left(X_{t}\right)-\phi^{-1}\left(Y_{t}\right)\right) \mathrm{d} b_{t}+J\left(X_{t}, Y_{t}\right) \mathrm{d} t
$$

up to the coupling time $\tau:=\inf \left\{t \geqslant 0: X_{t}=Y_{t}\right\}$, where $b_{t}$ is a one-dimensional Brownian motion. From this, it now suffices for us to estimate the term $I_{Z}^{\phi}$. Write $\rho^{\prime}=\rho^{\prime}(x, y)$ and for a minimizing $g^{\prime}$-geodesic $\gamma$ with $\gamma(0)=x$ and $\gamma\left(\rho^{\prime}\right)=y$ let

$$
J_{i}(s)=\phi^{-1}(\gamma(s)) P_{\gamma(0), \gamma(s)}^{\prime} V_{i}, \quad 1 \leqslant i \leqslant d
$$

where $J_{i}(0)=\phi^{-1}(x) V_{i}$ and $J_{i}\left(\rho^{\prime}\right)=\phi^{-1}(y) P_{x, y}^{\prime} V_{i}$. Since $P_{\gamma(0), \gamma(s)}^{\prime} V_{i}$ are parallel vector fields along $\gamma$ with respect to the metric $g^{\prime}$, we have that for $(x, y) \notin \operatorname{cut} \cup D(M)$, that

$$
\begin{aligned}
& \sum_{i=1}^{d}\left(U_{i}\right)^{2} \rho^{\prime}(x, y) \\
& \leqslant \sum_{i=1}^{d} \int_{0}^{\rho^{\prime}}\left\{\left|\nabla_{\dot{\gamma}}^{\prime} J_{i}\right|^{\prime 2}-\left\langle R^{\prime}\left(\dot{\gamma}, J_{i}\right) J_{i}, \dot{\gamma}\right\rangle^{\prime}\right\}(s) \mathrm{d} s \\
& =d \int_{0}^{\rho^{\prime}} \phi^{-2}(\gamma(s))\langle\nabla \log \phi(\gamma(s)), \dot{\gamma}(s)\rangle^{2} \mathrm{~d} s-\int_{0}^{\rho^{\prime}} \phi^{-2}(\gamma(s)) \operatorname{Ric}^{\prime}(\dot{\gamma}(s), \dot{\gamma}(s)) \mathrm{d} s .
\end{aligned}
$$

On the other hand

$$
\begin{aligned}
\phi^{-2}(x)\left\langle Z^{\prime}(x), \nabla^{\prime} \rho^{\prime}(\cdot, y)(x)\right\rangle^{\prime}+\phi^{-2}(y)\left\langle Z^{\prime}(y), \nabla^{\prime} \rho^{\prime}(x, \cdot)(y)\right\rangle^{\prime} \\
=\int_{0}^{\rho^{\prime}} \frac{\mathrm{d}}{\mathrm{d} s}\left\{\phi^{-2}(\gamma(s))\left\langle Z^{\prime}(\gamma(s)), \dot{\gamma}(s)\right\rangle^{\prime}\right\} \mathrm{d} s \\
=\int_{0}^{\rho^{\prime}} \phi^{-2}(\gamma(s))\left\langle\left(\nabla_{\dot{\gamma}}^{\prime} Z^{\prime}\right) \circ \gamma, \dot{\gamma}\right\rangle^{\prime}(s) \mathrm{d} s \\
\quad-2 \int_{0}^{\rho^{\prime}} \phi^{-2}(\gamma(s))\langle\nabla \log \phi(\gamma(s)), \dot{\gamma}(s)\rangle\left\langle Z^{\prime}(\gamma(s)), \dot{\gamma}(s)\right\rangle^{\prime} \mathrm{d} s .
\end{aligned}
$$

Moreover

$$
\left\langle Z^{\prime}(\gamma(s)), \dot{\gamma}(s)\right\rangle^{\prime}=\langle Z, \dot{\gamma}(s)\rangle+(d-2)\langle\nabla \log \phi, \dot{\gamma}(s)\rangle .
$$

Combining this with (2.5) and (2.6), we have

$$
\begin{aligned}
I_{Z}^{\phi}(x, y) \leqslant- & \int_{0}^{\rho^{\prime}} \phi^{-2}(\gamma(s))\left(\left(\operatorname{Ric}^{Z}\right)^{\prime}(\dot{\gamma}(s), \dot{\gamma}(s))+(d-4)\langle\nabla \log \phi, \dot{\gamma}(s)\rangle^{2} \mathrm{~d} s\right. \\
& \left.-\int_{0}^{\rho^{\prime}} 2\langle\nabla \log \phi, \dot{\gamma}(s)\rangle\langle Z, \dot{\gamma}(s)\rangle\right) \mathrm{d} s .
\end{aligned}
$$

By [3, Theorem 1.159], in which the Laplacian differs from our's by a negative sign, we know that

$$
\begin{aligned}
\left(\operatorname{Ric}^{Z}\right)^{\prime}(\dot{\gamma}, \dot{\gamma}) & =\operatorname{Ric}^{\prime}(\dot{\gamma}, \dot{\gamma})-\left\langle\nabla_{\dot{\gamma}}^{\prime} Z^{\prime}, \dot{\gamma}\right\rangle^{\prime} \\
& =\operatorname{Ric}^{Z}(\dot{\gamma}, \dot{\gamma})+\frac{1}{2} L \phi^{2}-2\langle\nabla \log \phi, \dot{\gamma}\rangle\langle Z, \dot{\gamma}\rangle-(d-2)\langle\dot{\gamma}, \nabla \log \phi\rangle^{2}-2|\nabla \phi|^{2}
\end{aligned}
$$

and, noting that $|\dot{\gamma}|=\phi$, we thus have

$$
\left(\operatorname{Ric}^{Z}\right)^{\prime}(\dot{\gamma}(s), \dot{\gamma}(s))+(d-4)\langle\nabla \log \phi, \dot{\gamma}(s)\rangle^{2}+2\langle\nabla \log \phi, \dot{\gamma}(s)\rangle\langle Z, \dot{\gamma}(s)\rangle
$$




$$
\begin{aligned}
& =\operatorname{Ric}^{Z}(\dot{\gamma}(s), \dot{\gamma}(s))+\frac{1}{2} L \phi^{2}-2\langle\dot{\gamma}, \nabla \log \phi\rangle^{2}-2|\nabla \phi|^{2} \\
& \geqslant \operatorname{Ric}^{Z}(\dot{\gamma}(s), \dot{\gamma}(s))+\frac{1}{2} L \phi^{2}-4|\nabla \phi|^{2} \\
& =\operatorname{Ric}^{Z}(\dot{\gamma}(s), \dot{\gamma}(s))+\phi^{2} L \log \phi-2|\nabla \phi|^{2} .
\end{aligned}
$$

Consequently, using the condition (2.1), letting $X_{t}=Y_{t}$ after coupling time, and then combining (2.7) with (2.8) and (2.4), we arrive at

$$
\mathrm{d} \rho^{\prime}\left(X_{t}, Y_{t}\right) \leqslant \sqrt{2}\left(\phi^{-1}\left(X_{t}\right)-\phi^{-1}\left(Y_{t}\right)\right) \mathrm{d} b_{t}-K_{\phi} \rho^{\prime}\left(X_{t}, Y_{t}\right) \mathrm{d} t .
$$

From this, we know that

$$
\mathbb{E}^{(x, y)}\left[\rho^{\prime}\left(X_{t}, Y_{t}\right)\right] \leqslant \mathrm{e}^{-K_{\phi} t} \rho^{\prime}(x, y) .
$$

Then, observing that $\rho^{\prime} \leqslant \rho \leqslant\|\phi\|_{\infty} \rho^{\prime}$, we have

$$
\begin{aligned}
\left|\nabla P_{t} f\right|(x) & =\lim _{y \rightarrow x}\left|\frac{P_{t} f(x)-P_{t} f(y)}{\rho(x, y)}\right| \\
& =\lim _{y \rightarrow x}\left|\mathbb{E}^{(x, y)}\left[\frac{f\left(X_{t}\right)-f\left(Y_{t}\right)}{\rho\left(X_{t}, Y_{t}\right)} \frac{\rho\left(X_{t}, Y_{t}\right)}{\rho^{\prime}\left(X_{t}, Y_{t}\right)} \frac{\rho^{\prime}\left(X_{t}, Y_{t}\right)}{\rho^{\prime}(x, y)} \frac{\rho^{\prime}(x, y)}{\rho(x, y)}\right]\right| \\
& \leqslant\|\phi\|_{\infty}\|\nabla f\|_{\infty} \mathrm{e}^{-K_{\phi} t}
\end{aligned}
$$

which completes the proof.

Remark 2.3. (i) Since $\left(U_{d}\right)^{2} \rho^{\prime} \neq 0$, it was indeed necessary to account for this quantity in inequality (2.5), correcting the proof of [16, Theorem 3.4.6].

(ii) Compared with the proof of [16, Theorem 3.4.6], our choice of vector field $J_{i}$ yields a simpler result.

(iii) In [17], a certain technical assumption which was used to ensure the uniformly boundedness of $|\nabla P . f|$ on $[0, t] \times M$ is no longer needed in the results.

The following results remove the additional condition in [16, Corollary 3.6.5 (1)] and [17, Corollary $1.2(1)]$ to ensure the uniform boundedness of $|\nabla P . f|$ on $[0, t] \times M$ and give a another proof of an extension of these inequalities to $L^{p}$ forms for $p>1$ :

Theorem 2.4. If there exists $\phi \in \mathscr{D}$ such that for $p>1$ the inequality

$$
\operatorname{Ric}^{Z}+L \log \phi-p|\nabla \log \phi|^{2} \geqslant K_{\phi, p}
$$

holds, then for $t>0$ and $f \in C_{b}^{1}(M)$,

$$
\left|\nabla P_{t} f\right| \leqslant \frac{1}{\phi} \mathrm{e}^{-K_{\phi, p} t}\left(P_{t}(\phi|\nabla f|)^{p /(p-1)}\right)^{(p-1) / p} .
$$

Proof. The lower bound (2.10) implies $\operatorname{Ric}^{Z}+L \log \phi-2|\nabla \log \phi|^{2}$ is bounded below (since $\phi \in \mathscr{D}$ implies $|\nabla \log \phi|$ bounded). By Theorem 2.2, it follows that $|\nabla P . f|$ is bounded on $[0, t] \times M$. Furthermore

$$
\operatorname{Ric}^{Z} \geqslant K_{\phi, p}-L \log \phi+p|\nabla \log \phi|^{2}=K_{\phi, p}+\frac{1}{p} \phi^{p} L \phi^{-p} \quad \text { and } \quad \text { II } \geqslant-N \log \phi
$$

and so, by Theorem 2.1, there exists $\left\{Q_{s}\right\}_{s \in[0, t]}$ such that

$$
\left\|Q_{t}\right\| \leqslant \exp \left(-K_{\phi, p} t-\frac{1}{p} \int_{0}^{t} \phi^{p} L \phi^{-p}\left(X_{s}\right) \mathrm{d} s+\int_{0}^{t} N \log \phi\left(X_{s}\right) \mathrm{d} l_{s}\right)
$$

with

$$
\left|\nabla P_{t} f\right|^{p} \leqslant\left(P_{t}(\phi|\nabla f|)^{p /(p-1)}\right)^{p-1} \mathbb{E}\left[\phi^{-p}\left(X_{t}\right)\left\|Q_{t}\right\|^{p}\right]
$$


It therefore suffices to give the upper bound estimate of the following term:

$$
\mathbb{E}\left[\phi^{-p}\left(X_{t}\right) \exp \left(-\int_{0}^{t} \phi^{p} L \phi^{-p}\left(X_{s}\right) \mathrm{d} s+p \int_{0}^{t} N \log \phi\left(X_{s}\right) \mathrm{d} l_{s}\right)\right] .
$$

To this end, by the Itô formula, it is easy to see that

$$
\begin{aligned}
\mathrm{d} \phi^{-p}\left(X_{t}\right) & =\left\langle\nabla \phi^{-p}\left(X_{t}\right), u_{t} \mathrm{~d} B_{t}\right\rangle+L \phi^{-p}\left(X_{t}\right) \mathrm{d} t+N \phi^{-p}\left(X_{t}\right) \mathrm{d} l_{t} \\
& =\left\langle\nabla \phi^{-p}\left(X_{t}\right), u_{t} \mathrm{~d} B_{t}\right\rangle-p \phi^{-p}\left(X_{t}\right)\left(-\frac{1}{p} \phi^{p} L \phi^{-p}\left(X_{t}\right) \mathrm{d} t+N \log \phi\left(X_{t}\right) \mathrm{d} l_{t}\right) .
\end{aligned}
$$

So

$$
M_{t}=\phi^{-p}\left(X_{t}\right) \exp \left(-\int_{0}^{t} \phi^{p}\left(X_{s}\right) L \phi^{-p}\left(X_{s}\right) \mathrm{d} s+p \int_{0}^{t} N \log \phi\left(X_{s}\right) \mathrm{d} l_{s}\right)
$$

is a positive local martingale. Thus

$$
\mathbb{E}\left[\phi^{-p}\left(X_{t}\right) \exp \left(-\int_{0}^{t} \phi^{p}\left(X_{s}\right) L \phi^{-p}\left(X_{s}\right) \mathrm{d} s+p \int_{0}^{t} N \log \phi\left(X_{s}\right) \mathrm{d} l_{s}\right)\right] \leqslant \phi^{-p}(x) .
$$

Combining this with (2.11) and (2.12) completes the proof.

Corollary 2.5. If there exists $\phi \in \mathscr{D}$ such that for $p>1$ the inequality

$$
\operatorname{Ric}^{Z}+L \log \phi-p|\nabla \log \phi|^{2} \geqslant K_{\phi, p}
$$

holds, then for $t>0$ and $f \in C_{b}^{1}(M)$,

$$
\left|\nabla P_{t} f\right| \leqslant\|\phi\|_{\infty} \mathrm{e}^{-K_{\phi, p} t}\left(P_{t}|\nabla f|^{p /(p-1)}\right)^{(p-1) / p} ;
$$

and for $f \in \mathscr{B}_{b}(M)$ and $t>0$,

$$
\left|\nabla P_{t} f\right|^{2} \leqslant\|\phi\|_{\infty}^{2} \frac{K_{\phi, 2}}{\mathrm{e}^{2 K_{\phi, 2} t}-1} P_{t} f^{2} .
$$

Proof. The first assertion follows from Theorem 2.4 by observing $\phi \geqslant 1$. As Theorem 2.1 can be used under our condition directly, the main idea of the proof of (2.13) is similar to that of [16, Corollary 3.2.8], so we skip it here.

Note that taking the limit $p \downarrow 1$ in Corollary 2.5 improves Theorem 2.2 by replacing the constant $K_{\phi}$ with $K_{\phi, 1}$.

\subsection{Harnack inequalities}

In [16, Theorem 3.4.7] and [14, Theorem 3.1], F.-Y. Wang used a coupling method to obtain dimension free Harnack inequalities and a log-Harnack inequality on manifolds with boundary, assuming $\operatorname{Ric}^{Z} \geqslant K$ for some $K \in C(M)$ with $\phi \in \mathscr{D}$ such that $\tilde{K}_{\phi}$ is finite (where the quantity $\tilde{K}_{\phi}$ is defined as in (1.1)). The coefficient involved in these inequalities is:

$$
2 \tilde{K}_{\phi}^{-}+4\|\phi Z+(d-2) \nabla \phi\|_{\infty}\|\nabla \log \phi\|_{\infty}+2 d\|\nabla \log \phi\|_{\infty}^{2} .
$$

We now give the following result, weakening the curvature condition, in terms of a different coefficient:

Theorem 2.6. Assume there exists $\phi \in \mathcal{D}$ such that

$$
\operatorname{Ric}^{Z}+L \log \phi-3|\nabla \log \phi|^{2} \geqslant K_{\phi, 3}
$$

for some constant $K_{\phi, 3}$. Then for $T>0, x, y \in M, p>\|\phi\|_{\infty}^{2}$ and $f \in C_{b}^{1}(M)$, we have

$$
\left(P_{T} f(y)\right)^{p} \leqslant P_{T} f^{p}(x) \exp \left(\frac{\sqrt{p}(\sqrt{p}-1) K_{\phi, 3}\|\phi\|_{\infty}^{2} \rho^{2}(x, y)}{8 \delta_{p}\left(\sqrt{p}-1-\delta_{p}\right)\left(\mathrm{e}^{2 K_{\phi, 3} T}-1\right)}\right),
$$

where $\delta_{p}=\max \left\{\|\phi\|_{\infty}-1, \frac{\sqrt{p}-1}{2}\right\}$. 
Proof. Fix $x, y \in M$ and $T>0$. As in the proof of Theorem 2.2, we consider the process $X_{t}$ generated by $L=\Delta+Z$ under the metric $g^{\prime}:=\phi^{-2} g$, for which the boundary $\left(\partial M, g^{\prime}\right)$ is convex. Let $X_{t}$ solve equation (2.3) with $X_{0}=x$. For a strictly positive function $\xi \in C([0, T))$, to be later determined, let $Y_{t}$ solve

$$
\begin{aligned}
\mathrm{d}_{I} Y_{t}= & \sqrt{2} \phi^{-1}\left(Y_{t}\right) 1_{\left\{\left(X_{t}, Y_{t}\right) \notin \mathrm{cut}\right\}} P_{X_{t}, Y_{t}}^{\prime} u_{t} \mathrm{~d} B_{t}+\sqrt{2} \phi^{-1}\left(Y_{t}\right) 1_{\left\{\left(X_{t}, Y_{t}\right) \in \operatorname{cut}\right\}} \tilde{u}_{t} \mathrm{~d} B_{t}^{\prime} \\
& +\phi_{t}^{-2} Z^{\prime}\left(Y_{t}\right) \mathrm{d} t-\frac{\phi^{-1}\left(Y_{t}\right) \rho^{\prime}\left(X_{t}, Y_{t}\right)}{\phi^{-1}\left(X_{t}\right) \xi(t)} \nabla^{\prime} \rho^{\prime}\left(X_{t}, \cdot\right)\left(Y_{t}\right) \mathrm{d} t+N^{\prime}\left(Y_{t}\right) \mathrm{d} \tilde{l}_{t}
\end{aligned}
$$

for $t \in[0, T)$, with $Y_{0}=y$, where $\tilde{l}_{t}$ is the local time of $Y_{t}$ on $\partial M, \tilde{u}_{t}$ is the lift process and $B_{t}^{\prime}$ is the Brownian motion independent of $B_{t}$ from earlier. In the following, we begin with the same argument as in the proof of [16, Theorem 3.4.7]. The different part is how to use our curvature condition to get a new estimate for the radial process $\rho^{\prime}\left(X_{t}, Y_{t}\right)$. To this end, as explained in the proof of Theorem 2.2, we may for the sake of conciseness disregard certain technical considerations relating to the cut locus of $M$. Consider the process $\left(X_{t}, Y_{t}\right)$ starting from $(x, y)$, which is a well defined continuous process for $t \leqslant T \wedge \zeta$ where $\zeta$ is the explosion time of $Y_{t}$; that is $\zeta:=\lim _{n \rightarrow \infty} \zeta_{n}$ for $\zeta_{n}:=\inf \left\{t>0: \rho^{\prime}\left(y, Y_{t}\right) \geqslant n\right\}$. Let

$$
\mathrm{d} \tilde{B}_{t}=\mathrm{d} B_{t}+\frac{\rho^{\prime}\left(X_{t}, Y_{t}\right)}{\sqrt{2} \xi(t) \phi^{-1}\left(X_{t}\right)} u_{t}^{-1} \nabla^{\prime} \rho^{\prime}\left(\cdot, Y_{t}\right)\left(X_{t}\right) \mathrm{d} t, \quad 0 \leqslant t<T \wedge \zeta .
$$

By the Girsanov theorem, for any $s \in(0, T)$ the process $B_{t}$ is a $d$-dimensional Brownian motion under the probability measure $R_{s} \mathbb{P}$ for

$$
R_{s}:=\exp \left[-\int_{0}^{s} \frac{\rho^{\prime}\left(X_{t}, Y_{t}\right)}{\xi(t) \phi^{-1}\left(X_{t}\right)}\left\langle\nabla^{\prime} \rho\left(\cdot, Y_{t}\right)\left(X_{t}\right), u_{t} \mathrm{~d} B_{t}\right\rangle^{\prime}-\frac{1}{2} \int_{0}^{s} \frac{\rho^{\prime}\left(X_{t}, Y_{t}\right)^{2}}{\xi^{2} \phi^{-2}\left(X_{t}\right)} \mathrm{d} t\right] .
$$

Let $\mathbb{Q}=R_{T \wedge \zeta} \mathbb{P}$. It has been shown in the proof of $[16$, Theorem 3.4.7] that $\mathbb{Q}(\zeta=T)=1$ and the coupling is successful up to the time $T$. In the following, we look at the processes under the new measure $\mathbb{Q}$. Then

$$
\begin{aligned}
\mathrm{d}_{I} X_{t} & =\sqrt{2} \phi^{-1}\left(X_{t}\right) u_{t} \mathrm{~d} \tilde{B}_{t}+\phi^{-2}\left(X_{t}\right) Z^{\prime}\left(X_{t}\right) \mathrm{d} t-\frac{\rho^{\prime}\left(X_{t}, Y_{t}\right)}{\xi(t)} \nabla^{\prime} \rho^{\prime}\left(X_{t}, \cdot\right)\left(Y_{t}\right) \mathrm{d} t+N^{\prime}\left(X_{t}\right) \mathrm{d} \tilde{l}_{t} ; \\
\mathrm{d}_{I} Y_{t} & =\sqrt{2} \phi^{-1}\left(Y_{t}\right) P_{X_{t}, Y_{t}}^{\prime} u_{t} \mathrm{~d} \tilde{B}_{t}+\phi^{-2}\left(Y_{t}\right) Z^{\prime}\left(Y_{t}\right) \mathrm{d} t+N^{\prime}\left(Y_{t}\right) \mathrm{d} \tilde{l}_{t}, \quad t \leqslant T .
\end{aligned}
$$

Since

$$
\operatorname{Ric}^{Z}+L \log \phi-2|\nabla \log \phi|^{2} \geqslant K_{\phi, 3}+|\nabla \log \phi|^{2}, \quad t \in[0, T],
$$

by a similar calculation as for $(2.8)$ and $(2.9)$ we find

$$
\begin{aligned}
\mathrm{d} \rho^{\prime}\left(X_{t}, Y_{t}\right) \leqslant & \sqrt{2}\left(\phi^{-1}\left(X_{t}\right)-\phi^{-1}\left(Y_{t}\right)\right)\left\langle\nabla^{\prime} \rho^{\prime}\left(\cdot, Y_{t}\right)\left(X_{t}\right), u_{t} \mathrm{~d} \tilde{B}_{t}\right\rangle^{\prime} \\
& -\left(\int_{0}^{\rho^{\prime}\left(X_{t}, Y_{t}\right)}\left(K_{\phi, 3}+|\nabla \log \phi|^{2}\right)(\gamma(s)) \mathrm{d} s\right) \mathrm{d} t-\frac{\rho^{\prime}\left(X_{t}, Y_{t}\right)}{\xi(t)} \mathrm{d} t, \quad 0 \leqslant t<T
\end{aligned}
$$

which implies

$$
\begin{aligned}
\mathrm{d} \frac{\rho^{\prime}\left(X_{t}, Y_{t}\right)^{2}}{\xi(t)} \leqslant & \frac{2 \sqrt{2}}{\xi(t)} \rho^{\prime}\left(X_{t}, Y_{t}\right)\left(\phi^{-1}\left(X_{t}\right)-\phi^{-1}\left(Y_{t}\right)\right)\left\langle\nabla^{\prime} \rho^{\prime}\left(\cdot, Y_{t}\right)\left(X_{t}\right), u_{t} \mathrm{~d} \tilde{B}_{t}\right\rangle^{\prime} \\
& -\frac{\rho^{\prime}\left(X_{t}, Y_{t}\right)^{2}}{\xi^{2}(t)}\left(\dot{\xi}(t)+2 K_{\phi, 3} \xi(t)+2\right) \mathrm{d} t, \quad 0 \leqslant t<T
\end{aligned}
$$

since the positive term coming from the covariation is smaller than the opposite of the negative term involving $|\nabla \log \phi|^{2}$. Now for $\theta \in(0,2)$ let

$$
\xi(t)=(2-\theta) \int_{t}^{T} \mathrm{e}^{-2 K_{\phi, 3}(t-s)} \mathrm{d} s, \quad t \in[0, T)
$$


so that $\xi$ solves the equation

$$
\dot{\xi}(t)+2 K_{\phi, 3} \xi(t)+2=\theta, \quad t \in[0, T) .
$$

Combining this with (2.17), we find

$$
\mathrm{d} \frac{\rho^{\prime}\left(X_{t}, Y_{t}\right)^{2}}{\xi(t)} \leqslant \frac{2 \sqrt{2}}{\xi(t)} \rho^{\prime}\left(X_{t}, Y_{t}\right)\left(\phi^{-1}\left(X_{t}\right)-\phi^{-1}\left(Y_{t}\right)\right)\left\langle\nabla^{\prime} \rho^{\prime}\left(\cdot, Y_{t}\right)\left(X_{t}\right), u_{t} \mathrm{~d} \tilde{B}_{t}\right\rangle^{\prime}-\frac{\rho^{\prime}\left(X_{t}, Y_{t}\right)^{2}}{\xi(t)^{2}} \theta \mathrm{d} t .
$$

The remainder of argument is given by the proof of [16, Theorem 3.4.7].

\subsection{Transportation-cost inequalities}

Consider $\mu, \nu \in \mathscr{P}(M)$ where $\mathscr{P}(M)$ denotes the space of all probability measures on $M$. Recall the $L^{p}$-Wasserstein distance between $\mu$ and $\nu$ is

$$
W_{p}(\mu, \nu)=\inf _{\eta \in \mathscr{C}(\mu, \nu)}\left\{\int_{M \times M} \rho(x, y)^{p} \mathrm{~d} \eta(x, y)\right\}^{1 / p}
$$

where $\mathscr{C}(\mu, \nu)$ is the set for couplings of $\mu$ and $\nu$. When the manifold has no boundary, it is well known that the curvature condition,

$$
\operatorname{Ric}^{Z} \geqslant K \text { for some constant } K
$$

is equivalent to

$$
W_{p}\left(\mu P_{t}, \nu P_{t}\right) \leqslant W_{p}(\mu, \nu) \mathrm{e}^{-K t}, \quad \mu, \nu \in \mathscr{P}(M),
$$

where $\mu P_{t} \in \mathscr{P}(M)$ is defined by $\left(\mu P_{t}\right)(A)=\mu\left(P_{t} 1_{A}\right)$ for measurable set $A$. This equivalence is due to [10] which is extended to the manifolds with convex boundary [15]. Using a coupling method, with the effect of the cut locus accommodated as in the proof of Theorem 2.6, we obtain the following transportation-cost inequality.

Theorem 2.7. If there exists $\phi \in \mathscr{D}$ and a constant $K_{\phi, 3}$ satisfying

$$
\operatorname{Ric}^{Z}+L \log \phi-3|\nabla \log \phi|^{2} \geqslant K_{\phi, 3}
$$

then

$$
W_{2}\left(\mu P_{t}, \nu P_{t}\right) \leqslant\|\phi\|_{\infty} \mathrm{e}^{-K_{\phi, 3} t} W_{2}(\mu, \nu) .
$$

Proof. By [16, Theorem 4.4.2], it suffices to only consider $\mu=\delta_{x}$ and $\nu=\delta_{y}$. Let $\phi$ be a smooth function in $\mathscr{D}$ and recall that $L=\phi^{-2}\left(\Delta^{\prime}+Z^{\prime}\right)$ for the manifold $\left(M, g^{\prime}\right)$ as in $(2.2)$, where $g^{\prime}=\phi^{-2} g$. Let $X_{t}$ and $Y_{t}$ solve the following SDEs respectively:

$$
\begin{aligned}
\mathrm{d}_{I} X_{t} & =\sqrt{2} \phi^{-1}\left(X_{t}\right) u_{t} \mathrm{~d} B_{t}+\phi^{-2}\left(X_{t}\right) Z^{\prime}\left(X_{t}\right) \mathrm{d} t+N^{\prime}\left(X_{t}\right) \mathrm{d} l_{t}, \quad X_{0}=x ; \\
\mathrm{d}_{I} Y_{t} & =\sqrt{2} \phi^{-1}\left(Y_{t}\right) P_{X_{t}, Y_{t}}^{\prime} u_{t} \mathrm{~d} B_{t}+\phi^{-2}\left(Y_{t}\right) Z^{\prime}\left(Y_{t}\right) \mathrm{d} t+N^{\prime}\left(Y_{t}\right) \mathrm{d} \tilde{l}_{t}, \quad Y_{0}=y .
\end{aligned}
$$

Then, as explained in the proof of Theorem 2.2, in which we derived (2.9), we have

$$
\begin{aligned}
\mathrm{d} \rho^{\prime}\left(X_{t}, Y_{t}\right) \leqslant & \sqrt{2}\left(\phi^{-1}\left(X_{t}\right)-\phi^{-1}\left(Y_{t}\right)\right)\left\langle\nabla^{\prime} \rho^{\prime}\left(\cdot, Y_{t}\right)\left(X_{t}\right), u_{t} \mathrm{~d} B_{t}\right\rangle^{\prime} \\
& -\left(\int_{0}^{\rho^{\prime}\left(X_{t}, Y_{t}\right)}\left(\phi^{-2} \operatorname{Ric}^{Z}(\dot{\gamma}(s), \dot{\gamma}(s))+L \log \phi-2|\nabla \log \phi|^{2}\right)(\gamma(s)) \mathrm{d} s\right) \mathrm{d} t .
\end{aligned}
$$

Therefore

$$
\begin{aligned}
\mathrm{d} \rho^{\prime}\left(X_{t}, Y_{t}\right)^{2}= & 2 \rho^{\prime}\left(X_{t}, Y_{t}\right) \mathrm{d} \rho^{\prime}\left(X_{t}, Y_{t}\right)+\left(\phi^{-1}\left(X_{t}\right)-\phi^{-1}\left(Y_{t}\right)\right)^{2} \mathrm{~d} t \\
\leqslant & \mathrm{~d} \tilde{M}_{t}+2\left(\int_{0}^{\rho^{\prime}\left(X_{t}, Y_{t}\right)}\left\langle\nabla^{\prime} \phi^{-1}(\gamma(s)), \dot{\gamma}(s)\right\rangle^{\prime} \mathrm{d} s\right)^{2} \mathrm{~d} t \\
& -2 \rho^{\prime}\left(X_{t}, Y_{t}\right) \int_{0}^{\rho^{\prime}\left(X_{t}, Y_{t}\right)}\left(\phi^{-2} \operatorname{Ric}^{Z}(\dot{\gamma}(s), \dot{\gamma}(s))+L \log \phi-2|\nabla \log \phi|^{2}\right)(\gamma(s)) \mathrm{d} s \mathrm{~d} t
\end{aligned}
$$




$$
\begin{aligned}
\leqslant & \mathrm{d} \tilde{M}_{t}-2 \rho^{\prime}\left(X_{t}, Y_{t}\right)\left(\int _ { 0 } ^ { \rho ^ { \prime } ( X _ { t } , Y _ { t } ) } \left(\phi^{-2}(\gamma(s)) \operatorname{Ric}^{Z}(\dot{\gamma}(s), \dot{\gamma}(s))\right.\right. \\
& \left.\left.+L \log \phi(\gamma(s))-3|\nabla \log \phi(\gamma(s))|^{2}\right) \mathrm{~d} s\right) \mathrm{d} t \\
\leqslant & \mathrm{~d} \tilde{M}_{t}-2 K_{\phi, 3} \rho^{\prime}\left(X_{t}, Y_{t}\right)^{2} \mathrm{~d} t,
\end{aligned}
$$

where

$$
\mathrm{d} \tilde{M}_{t}=2 \sqrt{2} \rho^{\prime}\left(X_{t}, Y_{t}\right)\left(\phi^{-1}\left(X_{t}\right)-\phi^{-1}\left(Y_{t}\right)\right)\left\langle\nabla^{\prime} \rho^{\prime}\left(\cdot, Y_{t}\right)\left(X_{t}\right), u_{t} \mathrm{~d} B_{t}\right\rangle^{\prime} .
$$

It follows that

$$
\begin{aligned}
W_{2}\left(\delta_{x} P_{t}, \delta_{y} P_{t}\right)^{2} & \leqslant \mathbb{E}^{(x, y)}\left[\rho\left(X_{t}, Y_{t}\right)^{2}\right] \leqslant\|\phi\|_{\infty}^{2} \mathbb{E}^{(x, y)}\left[\rho^{\prime}\left(X_{t}, Y_{t}\right)^{2}\right] \\
& \leqslant\|\phi\|_{\infty}^{2} \mathrm{e}^{-2 K_{\phi, 3} t} \rho^{\prime}(x, y)^{2} \leqslant\|\phi\|_{\infty}^{2} \mathrm{e}^{-2 K_{\phi, 3} t} \rho(x, y)^{2}
\end{aligned}
$$

which completes the proof.

We now investigate Talagrand-type inequalities with respect to the uniform distance on the path space $W^{T}:=C([0, T] ; M)$ of the (reflecting) diffusion process, for a given $T>0$. Let $X_{t}^{\mu}$ be the (reflecting if $\partial M \neq \varnothing)$ diffusion process generated by $L$ with initial distribution $\mu \in \mathscr{P}(M)$. Let $\Pi_{\mu}^{T}$ be the distribution of

$$
X_{[0, T]}^{\mu}:=\left\{X_{t}^{\mu}: t \in[0, T]\right\}
$$

which is a probability measure on the (free) path space $W^{T}$. When $\mu=\delta_{x}$ we denote $\Pi_{\delta_{x}}^{T}=\Pi_{x}^{T}$ and $X_{[0, T]}^{\delta_{x}}=X_{[0, T]}^{x}$. For any non-negative measurable function $F$ on $W^{T}$ such that $\Pi_{\mu}^{T}(F)=1$, one has

$$
\mu_{F}^{T}(\mathrm{~d} x):=\Pi_{x}^{T}(F) \mu(\mathrm{d} x) \in \mathscr{P}(M) .
$$

The the uniform distance on $W^{T}$ is given by

$$
\rho_{\infty}(\gamma, \eta):=\sup _{t \in[0, T]} \rho\left(\gamma_{t}, \eta_{t}\right), \quad \gamma, \eta \in W^{T} .
$$

Let $W_{2}^{\rho_{\infty}}$ be the $L^{2}$-Wasserstein distance (or $L^{2}$-transportation cost) induced by $\rho_{\infty}$. In general, for any $p \in[1, \infty)$ and two probability measures $\Pi_{1}, \Pi_{2}$ on $W^{T}$,

$$
W_{p}^{\rho_{\infty}}\left(\Pi_{1}, \Pi_{2}\right):=\inf _{\pi \in \mathscr{C}\left(\Pi_{1}, \Pi_{2}\right)}\left\{\iint_{W^{T} \times W^{T}} \rho_{\infty}(\gamma, \eta)^{p} \pi(\mathrm{d} \gamma, \mathrm{d} \eta)\right\}^{1 / p}
$$

is the $L^{p}$-Wasserstein distance (or $L^{p}$-transportation cost) of $\Pi_{1}$ and $\Pi_{2}$, induced by the uniform norm, where $\mathscr{C}\left(\Pi_{1}, \Pi_{2}\right)$ is the set of all couplings for $\Pi_{1}$ and $\Pi_{2}$. Moreover, for $F \geqslant 0$ with $\Pi_{\mu}^{T}(F)=1$, let

$$
\mu_{F}^{T}(\mathrm{~d} x)=\Pi_{x}^{T}(F) \mu(\mathrm{d} x) .
$$

The following result improves [15, Theorems 4.1 and 4.2] or [16, Theorems 4.5.3 and 4.5.4]:

Theorem 2.8. If there exists $\phi \in \mathscr{D}$ and a constant $K_{\phi}$ satisfying

$$
\operatorname{Ric}^{Z}+L \log \phi-2|\nabla \log \phi|^{2} \geqslant K_{\phi}
$$

then

(i) for $F \geqslant 0, \Pi_{\mu}^{T}(F)=1$ and $\mu \in \mathscr{P}(M)$,

$$
W_{2}^{\rho \infty}\left(F \Pi_{\mu}^{T}, \Pi_{\mu_{F}^{T}}^{T}\right)^{2} \leqslant \frac{2\|\phi\|_{\infty}^{2}}{K_{\phi}}\left(\mathrm{e}^{2 K_{\phi}^{+} T}-\mathrm{e}^{2 K_{\phi}^{-} T}\right) \inf _{R>0}\left\{\left(1+R^{-1}\right) \exp \left(8(1+R)\|\nabla \log \phi\|_{\infty}^{2}\right)\right\} \Pi_{\mu}^{T}(F \log F) ;
$$

(i') for $F \geqslant 0, \Pi_{\mu}^{T}(F)=1$ and $\mu \in \mathscr{P}(M)$,

$$
W_{2}^{\rho \infty}\left(F \Pi_{\mu}^{T}, \Pi_{\mu_{F}^{T}}^{T}\right)^{2} \leqslant \frac{2\|\phi\|_{\infty}^{2}}{K_{\phi}}\left(1-\mathrm{e}^{-2 K_{\phi} T}\right) \inf _{R>0}\left\{\left(1+R^{-1}\right) \exp \left(8(1+R)\|\nabla \log \phi\|_{\infty}^{2} \mathrm{e}^{2 K_{\phi}^{+} T}\right)\right\} \Pi_{\mu}^{T}(F \log F) ;
$$


(ii) for any $\mu, \nu \in \mathscr{P}(M)$,

$$
W_{2}^{\rho \infty}\left(\Pi_{\mu}^{T}, \Pi_{\nu}^{T}\right) \leqslant 2\|\phi\|_{\infty} \mathrm{e}^{\left(K_{\phi}^{-}+\|\nabla \log \phi\|_{\infty}\right) T} W_{2}(\mu, \nu)
$$

\section{Remark 2.9.}

(a) When $\|\nabla \log \phi\|_{\infty}>0$ and $K_{\phi}>0$ the upper bound in (i) is better than that in (i').

(b) When the boundary is convex we can choose $\phi \equiv 1$. In this case $\nabla \log \phi=0$ and the estimate in (i') is consistent with [16, Theorem 4.4.2 (2)] for the convex case.

(c) We note that $[16$, Theorem 4.4.2 (6)] needs to be corrected as follows:

$$
W_{2}^{\rho_{\infty}}\left(\Pi_{\mu}^{T}, \Pi_{\nu}^{T}\right) \leqslant \mathrm{e}^{K^{-} T} W_{2}(\mu, \nu),
$$

where $K$ is the lower bound of Ricci curvature. It is then consistent with Theorem 2.8 (ii) when $\phi \equiv 1$ and the boundary is convex.

Proof of Theorem 2.8.

(i) Simply denote $X_{[0, T]}^{x}=X_{[0, T]}$. Let $F$ be a positive bounded measurable function on $W^{T}$ such that $\inf F>0$ and $\Pi_{x}^{T}(F)=1$. Let

$$
\mathrm{d} \mathbb{Q}=F\left(X_{[0, T]}\right) \mathrm{d} \mathbb{P} .
$$

Since $\mathbb{E}\left[F\left(X_{[0, T]}\right)\right]=\Pi_{\mu}^{T}(F)=1, \mathbb{Q}$ is a probability measure on $\Omega$. Then we conclude that there exists a unique $\mathscr{F}_{t}$-predictable process $\beta_{t}$ on $\mathbb{R}^{d}$ such that

$$
F\left(X_{[0, T]}\right)=\exp \left(\int_{0}^{T}\left\langle\beta_{s}, \mathrm{~d} B_{s}\right\rangle-\frac{1}{2} \int_{0}^{T}\left\|\beta_{s}\right\|^{2} \mathrm{~d} s\right)
$$

and

$$
\int_{0}^{T} \mathbb{E}_{\mathbb{Q}}\left\|\beta_{s}\right\|^{2} \mathrm{~d} s=2 \mathbb{E}\left[F\left(X_{[0, T]}\right) \log F\left(X_{[0, T]}\right)\right] .
$$

Then, by the Girsanov theorem, $\tilde{B}_{t}:=B_{t}-\int_{0}^{t} \beta_{s} \mathrm{~d} s, t \in[0, T]$ is a $d$-dimensional Brownian motion under the probability measure $\mathbb{Q}$.

As explained in the proof of [16, Theorem 4.5.3], it suffices to assume $\mu=\delta_{x}, x \in M$. In this case, the desired inequality involves

$$
\mu_{F}^{T}=\delta_{x} \text { and } \Pi_{\mu}^{T}(F \log F)=\Pi_{x}^{T}(F \log F) .
$$

Since the diffusion coefficients are non-constant, it is convenient to adopt the Itô differential $\mathrm{d}_{I}$ for the Girsanov transformation. So the reflecting $L$ diffusion process $X_{t}$ can be constructed by solving the Itô SDE

$$
\mathrm{d}_{I} X_{t}=\sqrt{2} \phi^{-1}\left(X_{t}\right) u_{t} \mathrm{~d} B_{t}+\phi^{-2}\left(X_{t}\right) Z^{\prime}\left(X_{t}\right) \mathrm{d} t+N^{\prime}\left(X_{t}\right) \mathrm{d} l_{t}, \quad X_{0}=x,
$$

where $B_{t}$ is the $d$-dimensional Brownian motion with natural filtration $\mathscr{F}_{t}$. Then

$$
\mathrm{d}_{I} X_{t}=\sqrt{2} \phi^{-1}\left(X_{t}\right) u_{t} \mathrm{~d} \tilde{B}_{t}+\left\{\phi^{-2}\left(X_{t}\right) Z^{\prime}\left(X_{t}\right)+\sqrt{2} \phi^{-1}\left(X_{t}\right) u_{t} \beta_{t}\right\} \mathrm{d} t+N^{\prime}\left(X_{t}\right) \mathrm{d} l_{t}, \quad X_{0}=x
$$

and let $Y_{t}$ solve

$$
\mathrm{d}_{I} Y_{t}=\sqrt{2} \phi^{-1}\left(Y_{t}\right) P_{X_{t}, Y_{t}}^{\prime} u_{t} \mathrm{~d} \tilde{B}_{t}+\phi^{-2}\left(Y_{t}\right) Z^{\prime}\left(Y_{t}\right) \mathrm{d} t+N^{\prime}\left(Y_{t}\right) \mathrm{d} \tilde{l}_{t}, \quad Y_{0}=x
$$

where $l_{t}$ and $\tilde{l}_{t}$ are the local times of $X_{t}$ and $Y_{t}$ on $\partial M$, respectively. Moreover, for any bounded measurable function $G$ on $W^{T}$,

$$
\mathbb{E}_{\mathbb{Q}} G\left(X_{[0, T]}\right):=\mathbb{E}(F G)\left(X_{[0, T]}\right)=\Pi_{x}^{T}(F G) .
$$


We conclude that the distribution of $X_{[0, T]}$ under $\mathbb{Q}$ coincides with $F \Pi_{x}^{T}$. Therefore,

$$
\begin{aligned}
W_{2}^{\rho_{\infty}}\left(F \Pi_{x}^{T}, \Pi_{x}^{T}\right)^{2} & \leqslant \mathbb{E}_{\mathbb{Q}} \rho_{\infty}\left(X_{[0, T]}, Y_{[0, T]}\right)^{2}=\mathbb{E}_{\mathbb{Q}} \max _{t \in[0, T]} \rho\left(X_{t}, Y_{t}\right)^{2} \\
& \leqslant\|\phi\|_{\infty}^{2} \mathbb{E}_{\mathbb{Q}} \max _{t \in[0, T]} \rho^{\prime}\left(X_{t}, Y_{t}\right)^{2}
\end{aligned}
$$

Note that due to the convexity of the boundary,

$$
\left\langle N^{\prime}(x), \nabla^{\prime} \rho(\cdot, y)(x)\right\rangle^{\prime} \leqslant 0, \quad x \in \partial M .
$$

From this and equations (2.20) and (2.21), it follows that

$$
\mathrm{d} \rho^{\prime}\left(X_{t}, Y_{t}\right) \leqslant \sqrt{2}\left(\phi^{-1}\left(X_{t}\right)-\phi^{-1}\left(Y_{t}\right)\right)\left\langle\nabla^{\prime} \rho^{\prime}\left(\cdot, Y_{t}\right)\left(X_{t}\right), u_{t} \mathrm{~d} \tilde{B}_{t}\right\rangle^{\prime}-K_{\phi} \rho^{\prime}\left(X_{t}, Y_{t}\right) \mathrm{d} t+\sqrt{2}\left\|\beta_{t}\right\| \mathrm{d} t .
$$

Defining

$$
M_{t}:=\sqrt{2} \int_{0}^{t} \mathrm{e}^{K_{\phi} s}\left(\phi^{-1}\left(X_{s}\right)-\phi^{-1}\left(Y_{s}\right)\right)\left\langle\nabla^{\prime} \rho^{\prime}\left(\cdot, Y_{s}\right)\left(X_{s}\right), u_{s} \mathrm{~d} \tilde{B}_{s}\right\rangle^{\prime}
$$

we have

$$
\rho^{\prime}\left(X_{t}, Y_{t}\right) \leqslant \mathrm{e}^{-K_{\phi} t}\left(M_{t}+\sqrt{2} \int_{0}^{t} \mathrm{e}^{K_{\phi} s}\left\|\beta_{s}\right\| \mathrm{d} s\right), \quad t \in[0, T] .
$$

So to prove (i), we will estimate the function

$$
h_{t}=\mathbb{E}_{\mathbb{Q}} \max _{s \in[0, t]} \mathrm{e}^{2 K_{\phi} s} \rho^{\prime}\left(X_{s}, Y_{s}\right)^{2} .
$$

By the Doob inequality, for any $R>0$, we have

$$
\begin{aligned}
h_{t}: & =\mathbb{E}_{\mathbb{Q}} \max _{s \in[0, t]} \mathrm{e}^{2 K_{\phi} s} \rho^{\prime}\left(X_{s}, Y_{s}\right)^{2} \\
& \leqslant(1+R) \mathbb{E}_{\mathbb{Q}} \max _{s \in[0, t]} M_{s}^{2}+2\left(1+R^{-1}\right) \max _{s \in[0, t]} \mathbb{E}_{\mathbb{Q}}\left(\int_{0}^{s} \mathrm{e}^{K_{\phi} r}\left\|\beta_{r}\right\| \mathrm{d} r\right)^{2} \\
& \leqslant 4(1+R) \mathbb{E}_{\mathbb{Q}} M_{t}^{2}+2\left(1+R^{-1}\right) \int_{0}^{t} \mathrm{e}^{2 K_{\phi} s} \mathrm{~d} s \int_{0}^{t} \mathbb{E}_{\mathbb{Q}}\left\|\beta_{s}\right\|^{2} \mathrm{~d} s \\
& \leqslant 8(1+R)\|\nabla \log \phi\|_{\infty}^{2} \int_{0}^{t} h_{s} \mathrm{~d} s+2\left(1+R^{-1}\right) \int_{0}^{T} \mathrm{e}^{2 K_{\phi} s} \mathrm{~d} s \int_{0}^{T} \mathbb{E}_{\mathbb{Q}}\left\|\beta_{s}\right\|^{2} \mathrm{~d} s, \quad t \in[0, T] .
\end{aligned}
$$

Since $h_{0}=0$, by using the Gronwall inequality, this inequality further implies

$$
h_{T} \leqslant 2\left(1+R^{-1}\right) \exp \left(8(1+R)\|\nabla \log \phi\|_{\infty}^{2}\right) \int_{0}^{T} \mathrm{e}^{2 K_{\phi} s} \mathrm{~d} s \int_{0}^{T} \mathbb{E}_{\mathbb{Q}}\left\|\beta_{s}\right\|^{2} \mathrm{~d} s
$$

By (2.19) and (2.24) we thus have

$$
\mathbb{E}_{\mathbb{Q}} \max _{s \in[0, T]} \rho^{\prime}\left(X_{s}, Y_{s}\right)^{2} \leqslant 4\left(1+R^{-1}\right) \exp \left(8(1+R)\|\nabla \log \phi\|_{\infty}^{2}\right) \frac{\mathrm{e}^{2 K_{\phi}^{+} T}-\mathrm{e}^{2 K_{\phi}^{-} T}}{2 K_{\phi}} \Pi_{x}^{T}(F \log F) .
$$

(i') For this we use the function

$$
\tilde{h}_{t}=\mathrm{e}^{2 K_{\phi} t} \mathbb{E}_{\mathbb{Q}} \max _{s \in[0, t]} \rho^{\prime}\left(X_{s}, Y_{s}\right)^{2} .
$$

The inequality (2.23) should then be modified as follows:

$$
\begin{aligned}
\tilde{h}_{t} & :=\mathrm{e}^{2 K_{\phi} t} \mathbb{E}_{\mathbb{Q}} \max _{s \in[0, t]} \rho^{\prime}\left(X_{s}, Y_{s}\right)^{2} \\
& \leqslant \mathrm{e}^{2 K_{\phi} t}(1+R) \mathbb{E}_{\mathbb{Q}} \max _{s \in[0, t]} \mathrm{e}^{-2 K_{\phi} s} M_{s}^{2}+2 \mathrm{e}^{2 K_{\phi} t}\left(1+R^{-1}\right) \max _{s \in[0, t]} \mathbb{E}_{\mathbb{Q}}\left(\int_{0}^{s} \mathrm{e}^{-K_{\phi}(s-r)}\left\|\beta_{r}\right\| \mathrm{d} r\right)^{2}
\end{aligned}
$$




$$
\begin{aligned}
& \leqslant 4(1+R) \mathrm{e}^{2 K_{\phi}^{+} t} \mathbb{E}_{\mathbb{Q}} M_{t}^{2}+2\left(1+R^{-1}\right) \int_{0}^{t} \mathrm{e}^{2 K_{\phi} r} \mathrm{~d} r \int_{0}^{t} \mathbb{E}_{\mathbb{Q}}\left\|\beta_{s}\right\|^{2} \mathrm{~d} s \\
& \leqslant 8(1+R)\|\nabla \log \phi\|_{\infty}^{2} \mathrm{e}^{2 K_{\phi}^{+} T} \int_{0}^{t} \tilde{h}_{s} \mathrm{~d} s+2\left(1+R^{-1}\right) \int_{0}^{T} \mathrm{e}^{2 K_{\phi} r} \mathrm{~d} r \int_{0}^{T} \mathbb{E}_{\mathbb{Q}}\left\|\beta_{s}\right\|^{2} \mathrm{~d} s, \quad t \in[0, T] .
\end{aligned}
$$

Since $\tilde{h}_{0}=0$, this inequality implies

$$
\tilde{h}_{T} \leqslant 2\left(1+R^{-1}\right) \exp \left(8(1+R)\|\nabla \log \phi\|_{\infty}^{2} \mathrm{e}^{2 K_{\phi}^{+} T}\right) \int_{0}^{T} \mathrm{e}^{2 K_{\phi} s} \mathrm{~d} s \int_{0}^{T} \mathbb{E}_{\mathbb{Q}}\left\|\beta_{s}\right\|^{2} \mathrm{~d} s .
$$

We then conclude that

$$
\mathbb{E}_{\mathbb{Q}} \max _{s \in[0, T]} \rho^{\prime}\left(X_{s}, Y_{s}\right)^{2} \leqslant 4\left(1+R^{-1}\right) \exp \left(8(1+R)\|\nabla \log \phi\|_{\infty}^{2} \mathrm{e}^{2 K_{\phi}^{+} T}\right) \frac{1-\mathrm{e}^{-2 K_{\phi} T}}{2 K_{\phi}} \Pi_{x}^{T}(F \log F) .
$$

(ii) Without loss of generality, we consider $\mu=\delta_{x}$, and $\nu=\delta_{y}$. Let $X_{t}$ and $Y_{t}$ solve the following SDEs, respectively:

$$
\begin{aligned}
& \mathrm{d}_{I} X_{t}=\sqrt{2} \phi^{-1}\left(X_{t}\right) u_{t} \mathrm{~d} B_{t}+\phi^{-2}\left(X_{t}\right) Z^{\prime}\left(X_{t}\right) \mathrm{d} t+N^{\prime}\left(X_{t}\right) \mathrm{d} l_{t}, \quad X_{0}=x ; \\
& \mathrm{d}_{I} Y_{t}=\sqrt{2} \phi^{-1}\left(Y_{t}\right) P_{X_{t}, Y_{t}}^{\prime} u_{t} \mathrm{~d} B_{t}+\phi^{-2}\left(Y_{t}\right) Z^{\prime}\left(Y_{t}\right) \mathrm{d} t+N^{\prime}\left(Y_{t}\right) \mathrm{d} \tilde{l}_{t}, \quad Y_{0}=y .
\end{aligned}
$$

Then, as explained in the proof of Theorem 2.2, we have

$$
\begin{aligned}
\mathrm{d} \rho^{\prime}\left(X_{t}, Y_{t}\right) \leqslant & \sqrt{2}\left(\phi^{-1}\left(X_{t}\right)-\phi^{-1}\left(Y_{t}\right)\right)\left\langle\nabla^{\prime} \rho^{\prime}\left(\cdot, Y_{t}\right)\left(X_{t}\right), u_{t} \mathrm{~d} B_{t}\right\rangle^{\prime} \\
& -\left(\int_{0}^{\rho^{\prime}\left(X_{t}, Y_{t}\right)}\left(\phi^{-2} \operatorname{Ric}^{Z}(\dot{\gamma}(s), \dot{\gamma}(s))+L \log \phi-2|\nabla \log \phi|^{2}\right)(\gamma(s)) \mathrm{d} s\right) \mathrm{d} t .
\end{aligned}
$$

Therefore,

$$
\rho^{\prime}\left(X_{t}, Y_{t}\right) \leqslant \mathrm{e}^{-K_{\phi} t}\left(\hat{M}_{t}+\rho^{\prime}(x, y)\right), \quad t \geqslant 0
$$

for

$$
\hat{M}_{t}:=\sqrt{2} \int_{0}^{t} \mathrm{e}^{K_{\phi} s}\left(\phi^{-1}\left(X_{s}\right)-\phi^{-1}\left(Y_{s}\right)\right)\left\langle\nabla^{\prime} \rho\left(\cdot, Y_{s}\right)\left(X_{s}\right), u_{s} \mathrm{~d} B_{s}\right\rangle^{\prime} .
$$

Again using the Itô formula, we have

$$
\mathrm{d} \rho^{\prime}\left(X_{t}, Y_{t}\right)^{2} \leqslant \mathrm{~d} \tilde{M}_{t}-2\left(K_{\phi}-\|\nabla \log \phi\|_{\infty}^{2}\right) \rho^{\prime}\left(X_{t}, Y_{t}\right)^{2} \mathrm{~d} t
$$

where

$$
\mathrm{d} \tilde{M}_{t}=2 \rho^{\prime}\left(X_{t}, Y_{t}\right)\left(\phi^{-1}\left(X_{t}\right)-\phi^{-1}\left(Y_{t}\right)\right)\left\langle\nabla^{\prime} \rho^{\prime}\left(\cdot, Y_{t}\right)\left(X_{t}\right), u_{t} \mathrm{~d} B_{t}\right\rangle^{\prime}
$$

which implies

$$
\mathbb{E} \rho^{\prime}\left(X_{t}, Y_{t}\right)^{2} \leqslant \mathrm{e}^{-2\left(K_{\phi}-\|\nabla \log \phi\|_{\infty}^{2}\right) t} \rho^{\prime}(x, y)^{2} .
$$

Combining this with (2.26) we arrive at

$$
\begin{aligned}
W_{2}^{\rho_{\infty}}\left(\Pi_{x}^{T}, \Pi_{y}^{T}\right)^{2} & \leqslant\|\phi\|_{\infty}^{2} \mathbb{E} \max _{t \in[0, T]} \rho^{\prime}\left(X_{t}, Y_{t}\right)^{2} \\
& \leqslant\|\phi\|_{\infty}^{2} \mathrm{e}^{2 K_{\phi}^{-} T} \mathbb{E} \max _{t \in[0, T]}\left(\hat{M}_{t}+\rho^{\prime}(x, y)\right)^{2} \\
& \leqslant 4\|\phi\|_{\infty}^{2} \mathrm{e}^{2 K_{\phi}^{-} T} \mathbb{E}\left(\hat{M}_{T}+\rho^{\prime}(x, y)\right)^{2} \\
& =4\|\phi\|_{\infty}^{2} \mathrm{e}^{2 K_{\phi}^{-} T}\left(\mathbb{E} \hat{M}_{T}^{2}+\rho^{\prime}(x, y)^{2}\right) \\
& \leqslant 4\|\phi\|_{\infty}^{2} \mathrm{e}^{2 K_{\phi}^{-} T}\left(2 \int_{0}^{T} \mathrm{e}^{2 K_{\phi} t}\|\nabla \log \phi\|_{\infty}^{2} \mathbb{E} \rho^{\prime}\left(X_{t}, Y_{t}\right)^{2} \mathrm{~d} t+\rho^{\prime}(x, y)^{2}\right)
\end{aligned}
$$




$$
\begin{aligned}
& \leqslant 4\|\phi\|_{\infty}^{2} \mathrm{e}^{2\left(K_{\phi}^{-}+\|\nabla \log \phi\|_{\infty}^{2}\right) T} \rho^{\prime}(x, y)^{2} \\
& \leqslant 4\|\phi\|_{\infty}^{2} \mathrm{e}^{2\left(K_{\phi}^{-}+\|\nabla \log \phi\|_{\infty}^{2}\right) T} \rho(x, y)^{2}
\end{aligned}
$$

where the second inequality is due to the Doob inequality. This implies the desired inequality for $\mu=\delta_{x}$ and $\nu=\delta_{y}$.

Corollary 2.10. If there exists $\phi \in \mathscr{D}$ and a constant $K_{\phi}$ satisfying

$$
\operatorname{Ric}^{Z}+L \log \phi-2|\nabla \log \phi|^{2} \geqslant K_{\phi}
$$

then

(i) for $F \geqslant 0, \Pi_{\mu}^{T}(F)=1$ and $\mu \in \mathscr{P}(M)$,

$$
W_{2}^{\rho_{\infty}}\left(F \Pi_{\mu}^{T}, \Pi_{\mu_{F}^{T}}^{T}\right)^{2} \leqslant \frac{2\|\phi\|_{\infty}^{2}}{K_{\phi}}\left(\mathrm{e}^{2 K_{\phi}^{+} T}-\mathrm{e}^{2 K_{\phi}^{-} T}\right) \exp \left(8\|\nabla \log \phi\|_{\infty}^{2}+4 \sqrt{2}\|\nabla \log \phi\|_{\infty}\right) \Pi_{\mu}^{T}(F \log F) ;
$$

(i') for $F \geqslant 0, \Pi_{\mu}^{T}(F)=1$ and $\mu \in \mathscr{P}(M)$,

$$
W_{2}^{\rho \infty}\left(F \Pi_{\mu}^{T}, \Pi_{\mu_{F}^{T}}^{T}\right)^{2} \leqslant \frac{2\|\phi\|_{\infty}^{2}}{K_{\phi}}\left(1-\mathrm{e}^{-2 K_{\phi} T}\right) \exp \left(8\|\nabla \log \phi\|_{\infty}^{2} \mathrm{e}^{2 K_{\phi}^{+} T}+4 \sqrt{2}\|\nabla \log \phi\|_{\infty} \mathrm{e}^{K_{\phi}^{+} T}\right) \Pi_{\mu}^{T}(F \log F) .
$$

Proof. It is easily observed that

$$
\left(1+R^{-1}\right) \exp \left(8(1+R)\|\nabla \log \phi\|_{\infty}^{2}\right) \leqslant \exp \left(R^{-1}+8(1+R)\|\nabla \log \phi\|_{\infty}^{2}\right) .
$$

Taking the infimum about $R$ on the right side above, we arrive at

$$
\exp \left(R^{-1}+8(1+R)\|\nabla \log \phi\|_{\infty}^{2}\right) \geqslant \exp \left(8\|\nabla \log \phi\|_{\infty}^{2}+4 \sqrt{2}\|\nabla \log \phi\|_{\infty}\right)
$$

which allows to prove (i). The inequality (i') can be checked in the same way.

\section{New construction of function $\log \phi$}

In this section, we give a new construction of a function $\phi$ which satisfies the conditions of the previous section. To do so, we let $\rho_{\partial}$ be the Riemannian distance to the boundary $\partial M$ and use a comparison theorem for $\Delta \rho_{\partial}$ near the boundary, essentially due to [8]. Note that, by using local charts, it is clear that $\rho_{\partial}$ is smooth in a neighbourhood of $\partial M$. We call

$$
i_{\partial}:=\sup \left\{r>0: \rho_{\partial} \text { is smooth on }\left\{\rho_{\partial}<r\right\}\right\}
$$

the injectivity radius of $\partial M$. Obviously, $i_{\partial}>0$ if $M$ is compact, but it could be zero in the non-compact case $(\sup \varnothing=0$ by convention). As [16, Theorem 1.2.3] we have:

Lemma 3.1. Let $\theta, k$ be constants such that $\mathrm{II} \leqslant \theta$ and Sect $\leqslant k$. Let

$$
h(t):= \begin{cases}\cos \sqrt{k} t-\frac{\theta}{\sqrt{k}} \sin \sqrt{k} t, & k>0, \\ 1-\theta t, & k=0, \\ \cosh \sqrt{-k} t-\frac{\theta}{\sqrt{-k}} \sinh \sqrt{-k} t, & k<0\end{cases}
$$

for $t \geqslant 0$. Let $h^{-1}(0)$ be the first zero of $h\left(\right.$ with $h^{-1}(0):=\infty$ if $h(t)>0$ for all $\left.t \geqslant 0\right)$. Then for any

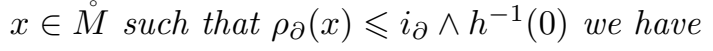

$$
\Delta \rho_{\partial}(x) \geqslant(d-1) \frac{h^{\prime}}{h}\left(\rho_{\partial}(x)\right)
$$


Note that if $k$ is positive then

$$
h^{-1}(0)=\frac{1}{\sqrt{k}} \arcsin \left(\sqrt{\frac{k}{k+\theta^{2}}}\right) .
$$

We now work under the following assumption:

Assumption (A) There exist non-negative constants $\sigma$ and $\theta$ such that $-\sigma \leqslant$ II $\leqslant \theta$ and a positive constant $r_{0}$ such that on $\partial_{r_{0}} M:=\left\{x \in M: \rho_{\partial}(x) \leqslant r_{0}\right\}$ the function $\rho_{\partial}$ is smooth, the norm of $Z$ is bounded and Sect $\leqslant k$ for some positive constant $k$.

Using this assumption, F.-Y. Wang constructed a function $\phi$ satisfying $\phi \in \mathscr{D}$ (see [13, p.1436] or [16, Theorem 3.2.9]). Following his construction, we define

$$
\log \phi(x)=\frac{\sigma}{\alpha} \int_{0}^{\rho_{\partial}(x)}\left[h(s)-h\left(r_{1}\right)\right]^{1-d} \mathrm{~d} s \int_{s \wedge r_{1}}^{r_{1}}\left[h(u)-h\left(r_{1}\right)\right]^{d-1} \mathrm{~d} u,
$$

where $r_{1}:=r_{0} \wedge h^{-1}(0)$ and

$$
\alpha:=\left(1-h\left(r_{1}\right)\right)^{1-d} \int_{0}^{r_{1}}\left[h(s)-h\left(r_{1}\right)\right]^{d-1} \mathrm{~d} s .
$$

Then from the proof of [12, Theorem 1.1], we know:

Theorem 3.2. Suppose that Assumption (A) holds and $\operatorname{Ric}^{Z} \geqslant K$. Define

$$
K_{p}=K-\sigma\left(\delta_{r_{1}}(Z)+\frac{d}{r_{1}}\right)-p \sigma^{2}
$$

where

$$
\delta_{r_{1}}(Z):=\sup \left\{|Z(x)|: x \in \partial_{r_{1}} M\right\} .
$$

Then all results in Section 2 hold by replacing

$$
K_{\phi}, K_{\phi, p},\|\phi\|_{\infty} \text { and }\|\nabla \log \phi\|_{\infty}
$$

with

$$
K_{2}, K_{p}, \mathrm{e}^{\sigma d r_{1} / 2} \text { and } \sigma
$$

respectively.

In the following we give a new construction of $\phi$ by using the function

$$
\ell(r)= \begin{cases}\mathrm{e}^{-2}-\mathrm{e}^{-2(1-2 r)^{-1}}, & 0 \leqslant r<\frac{1}{2} \\ \mathrm{e}^{-2}, & r \geqslant \frac{1}{2} .\end{cases}
$$

Proposition 3.3. Suppose that Assumption (A) holds. Let

$$
H(r):=\frac{\sqrt{k+\theta^{2}}}{k} \cos \left(\arcsin \left(\sqrt{\frac{k}{k+\theta^{2}}}\right)-\sqrt{k}\left(r \wedge r_{1}\right)\right)-\frac{\theta}{k} .
$$

Then the function

$$
\log \phi(x):=\frac{1}{2} \sigma \mathrm{e}^{2} \ell\left(\frac{H\left(\rho_{\partial}(x)\right)}{2 H\left(r_{1}\right)}\right) H\left(r_{1}\right)
$$

satisfies

$$
\left.N \log \phi\right|_{\partial M}=\sigma \geqslant- \text { II. }
$$

Moreover,

$$
\|\phi\|_{\infty} \leqslant \mathrm{e}^{\sigma H\left(r_{1}\right) / 2}, \quad|\nabla \log \phi| \leqslant \sigma
$$

and

$$
L \log \phi(x) \geqslant-\sigma\left(d \sqrt{\theta^{2}+k}+\delta_{r_{1}}(Z)+\frac{5}{2 H\left(r_{1}\right)}\right) .
$$


Proof of Proposition 3.3. First it is easy to see that the function $\ell$ satisfies $\ell \leqslant \mathrm{e}^{-2}$. Differentiating $\ell$ we obtain

$$
\ell^{\prime}(r)= \begin{cases}\left(\frac{1}{2}-r\right)^{-2} \mathrm{e}^{-\left(\frac{1}{2}-r\right)^{-1}}, & 0 \leqslant r<\frac{1}{2} \\ 0, & r \geqslant \frac{1}{2}\end{cases}
$$

and

$$
\ell^{\prime \prime}(r)= \begin{cases}-2 r\left(\frac{1}{2}-r\right)^{-4} \mathrm{e}^{-\left(\frac{1}{2}-r\right)^{-1}}, & 0 \leqslant r<\frac{1}{2} \\ 0, & r \geqslant \frac{1}{2} .\end{cases}
$$

As $\ell^{\prime \prime}<0$ on $[0,1 / 2)$, the function $\ell^{\prime}$ is at its maximal point when $r=0$, which implies $0 \leqslant \ell^{\prime} \leqslant 4 \mathrm{e}^{-2}$. Using the same method, when $r=\sqrt{3} / 6$ the function $\ell^{\prime \prime}$ reaches the minimal value, which implies

$$
\ell^{\prime \prime} \geqslant-3^{-1 / 2}(3+\sqrt{3})^{4} \mathrm{e}^{-(3+\sqrt{3})}>-20 \mathrm{e}^{-2} .
$$

Using these results, we have

$$
\left.N \log \phi\right|_{\partial M}=\frac{1}{4} \mathrm{e}^{2} \sigma \ell^{\prime}(0) N \rho_{\partial}=\sigma
$$

and

$$
|\nabla \log \phi|=\frac{1}{4} \mathrm{e}^{2} \sigma \ell^{\prime}\left(\frac{H\left(\rho_{\partial}\right)}{2 H\left(r_{0}\right)}\right) H^{\prime}\left(\rho_{\partial}\right) \leqslant \sigma .
$$

Moreover, by Lemma 3.1, we have

$$
\begin{aligned}
L \log \phi & =\frac{1}{4} \mathrm{e}^{2} \sigma\left(\ell^{\prime}\left(\frac{H\left(\rho_{\partial}\right)}{2 H\left(r_{0}\right)}\right) h\left(\rho_{\partial}\right) L \rho_{\partial}+\ell^{\prime \prime}\left(\frac{H\left(\rho_{\partial}\right)}{2 H\left(r_{0}\right)}\right) \frac{h\left(\rho_{\partial}\right)^{2}}{2 H\left(r_{0}\right)}+\ell^{\prime}\left(\frac{H\left(\rho_{\partial}\right)}{2 H\left(r_{0}\right)}\right) h^{\prime}\left(\rho_{\partial}(x)\right)\right) \\
& \geqslant \frac{1}{4} \mathrm{e}^{2} \sigma\left(\ell^{\prime}\left(\frac{H\left(\rho_{\partial}\right)}{2 H\left(r_{0}\right)}\right)\left(d h^{\prime}\left(\rho_{\partial}\right)-\sup _{\partial_{r_{0}} M}|Z|\right)+\frac{h\left(\rho_{\partial}\right)^{2}}{2 H\left(r_{0}\right)} \ell^{\prime \prime}\left(\frac{H\left(\rho_{\partial}\right)}{2 H\left(r_{0}\right)}\right)\right),
\end{aligned}
$$

where $h$ is defined as in (3.1) for $k \geqslant 0$. It is easy to calculate that

$$
h^{\prime}(r) \geqslant-\sqrt{\theta^{2}+k}
$$

Combining this with properties of $\ell$, we conclude that

$$
L \log \phi \geqslant-\sigma\left(d \sqrt{\theta^{2}+k}+\sup \left\{|Z|(x): x \in \partial_{r_{0} \wedge h^{-1}(0)} M\right\}+\frac{5}{2 H\left(r_{0}\right)}\right)
$$

which completes the proof.

Corollary 3.4. Suppose that Assumption (A) holds and $\operatorname{Ric}^{Z} \geqslant K$. Define

$$
\tilde{K}=K-\sigma\left(d \sqrt{\theta^{2}+k}+\delta_{r_{1}}(Z)+\frac{5}{2 H\left(r_{1}\right)}\right),
$$

and $\tilde{K}_{p}=\tilde{K}-p \sigma^{2}$ with $\delta_{r_{1}}(Z)$ as defined in (3.3). Then all results in Section 2 hold by replacing

$$
K_{\phi, p},\|\phi\|_{\infty} \text { and }\|\nabla \log \phi\|_{\infty}
$$

with

$$
\tilde{K}_{p}, \mathrm{e}^{\sigma H\left(r_{1}\right) / 2} \text { and } \sigma
$$

respectively.

Acknowledgements This work has been supported by Fonds National de la Recherche Luxembourg (Open project O14/7628746 GEOMREV), and partially by PUL AGSDE of the University of Luxembourg. The first named author acknowledges support by NSFC (Grant No. 11501508) and Zhejiang Provincial Natural Science Foundation of China (Grant No. LQ16A010009). 


\section{References}

1 Marc Arnaudon and Xue-Mei Li, Reflected Brownian motion: selection, approximation and linearization, Electron. J. Probab. 22 (2017), Paper No. 31, 55.

2 Marc Arnaudon, Anton Thalmaier and Feng-Yu Wang, Harnack inequality and heat kernel estimates on manifolds with curvature unbounded below, Bull. Sci. Math. 130 (2006), no. 3, 223-233.

3 Arthur L. Besse, Einstein manifolds, Ergebnisse der Mathematik und ihrer Grenzgebiete (3) [Results in Mathematics and Related Areas (3)], vol. 10, Springer-Verlag, Berlin, 1987.

4 Jean-Michel Bismut, Large deviations and the Malliavin calculus, Progress in Mathematics, vol. 45, Birkhäuser Boston, Inc., Boston, MA, 1984.

5 K. D. Elworthy and X.-M. Li, Formulae for the derivatives of heat semigroups, J. Funct. Anal. 125 (1994), no. 1, $252-286$.

6 Michel Émery, Stochastic calculus in manifolds, Universitext, Springer-Verlag, Berlin, 1989, With an appendix by P.-A. Meyer.

7 Elton P. Hsu, Multiplicative functional for the heat equation on manifolds with boundary, Michigan Math. J. 50 (2002), no. 2, 351-367.

8 Atsushi Kasue, A Laplacian comparison theorem and function theoretic properties of a complete Riemannian manifold, Japan. J. Math. (N.S.) 8 (1982), no. 2, 309-341.

9 Anton Thalmaier, On the differentiation of heat semigroups and Poisson integrals, Stochastics Stochastics Rep. 61 (1997), no. 3-4, 297-321.

10 Max-K. von Renesse and Karl-Theodor Sturm, Transport inequalities, gradient estimates, entropy, and Ricci curvature, Comm. Pure Appl. Math. 58 (2005), no. 7, 923-940.

11 Feng-Yu Wang, Functional inequalities, Markov semigroups and spectral theory, Science Press, 16 Donghuangchenggen North Street, Beijing 100717, China, 2005.

12 Feng-Yu Wang, Gradient estimates and the first Neumann eigenvalue on manifolds with boundary, Stochastic Process. Appl. 115 (2005), no. 9, 1475-1486.

13 Feng-Yu Wang, Estimates of the first Neumann eigenvalue and the log-Sobolev constant on non-convex manifolds, Math. Nachr. 280 (2007), no. 12, 1431-1439.

14 Feng-Yu Wang, Harnack inequality for SDE with multiplicative noise and extension to Neumann semigroup on nonconvex manifolds, Ann. Probab. 39 (2011), no. 4, 1449-1467.

15 Feng-Yu Wang, Transportation-cost inequalities on path space over manifolds with boundary, Doc. Math. 18 (2013), $297-322$.

16 Feng-Yu Wang, Analysis for diffusion processes on Riemannian manifolds, Advanced Series on Statistical Science \& Applied Probability, 18, World Scientific Publishing Co. Pte. Ltd., Hackensack, NJ, 2014.

17 Feng-Yu Wang, Modified curvatures on manifolds with boundary and applications, Potential Anal. 41 (2014), no. 3, 699-714. 